\title{
ELECTRON-POSITRON CORRELATIONS IN PARAMAGNETIC CHROMIUM
}

\author{
A. RubaszeK ${ }^{a}$, Z. SzoteK ${ }^{b}$ and W.M. Temmerman ${ }^{b}$ \\ ${ }^{a}$ W. Trzebiatowski Institute of Low Temperatures and Structure Research \\ Polish Academy of Sciences, P.O. Box 1410, 50-950 Wrocław 2, Poland \\ ${ }^{b}$ Daresbury Laboratory, Daresbury, Warrington, WA4 4AD, Cheshire, UK
}

\begin{abstract}
Several approximations for the electron-positron interaction are applied to study the electron-positron momentum densities of paramagnetic chromium. The non-local and state selective electron-positron correlation functions, evaluated within the weighted density approximation, are used to calculate various positron annihilation characteristics in this system. The weighted density approximation results are compared to the experimental data, and to the corresponding results of the independent particle model, local density approximation, and generalised gradient approximation. Also, the semi-empirical results are used in gaining more physical insight and deriving final conclusions of this work.
\end{abstract}

PACS numbers: 78.70.Bj, 71.10.-w, 71.60.+z

The detailed knowledge of the Fermi surface (FS) of chromium [1-5] is of great importance for the understanding of its physical properties. Chromium becomes antiferromagnetic at low temperatures $\left(T_{\mathrm{N}}=311 \mathrm{~K}\right)$, and the associated spin density wave is incommensurate with the lattice with a period that is inversely proportional to the length of the FS nesting vectors. Recently, giant magnetoresistance has been discovered in $\mathrm{Fe} / \mathrm{Cr}$ multilayers, and the period of the intralayer exchange coupling is proportional to the FS nesting vectors of the Cr spacer layer. These rich FS derived properties of $\mathrm{Cr}$ have motivated new positron annihilation studies [6], and the purpose of the present work is to contribute to the understanding of the positron annihilation characteristics of $\mathrm{Cr}$, measured by positron annihilation techniques.

Positron annihilation experiment is not restricted to very low temperatures or to samples of high purity, and the interpretation of angular correlation of annihilation radiation (ACAR) spectra depends only on the conservation of momentum and energy of the annihilating electron-positron (e-p) pair [7]. The experimental 
ACAR spectra are usually identified with projections of the e-p momentum density

$$
\rho(p=k+\boldsymbol{G})=\sum_{j}^{\text {occ }}\left|\int \exp (-\mathrm{i} \boldsymbol{p} \cdot \boldsymbol{r}) \psi_{\boldsymbol{k} j}(\boldsymbol{r}) \varphi_{+}(\boldsymbol{r}) \sqrt{\gamma(\boldsymbol{k j}, \boldsymbol{r})} \mathrm{d} \boldsymbol{r}\right|^{2},
$$

where $\boldsymbol{G}$ denotes the reciprocal lattice vector, $\boldsymbol{k}$ is the momentum in the first Brillouin zone (BZ), and the summation runs over all occupied electron bands $j$. Here $\psi_{\boldsymbol{k}_{j}}$ and $\varphi_{+}$are respectively the wave functions of an electron in the initial Bloch state $\boldsymbol{k} j$ and a thermalised positron. The quantities $\gamma(\boldsymbol{k} j, \boldsymbol{r})$ are the two-particle $\mathrm{e}^{-} \mathrm{p}$ correlation functions which, in general, depend both on the electron state $\boldsymbol{k} j$ and positron position $r$.

In the present work we calculate the positron annihilation characteristics of the paramagnetic chromium within the non-local approach of the weighted density approximation (WDA) [8] to the e-p correlation functions, $\gamma(\boldsymbol{k} j, \boldsymbol{r})$, in Eq. (1). The WDA results are compared to those obtained within the independent particle model (IPM), local density approximation (LDA) [9], non-local generalised gradient approximation (GGA) [10], the results of a semi-empirical analysis [5], as well as experimental data [4].

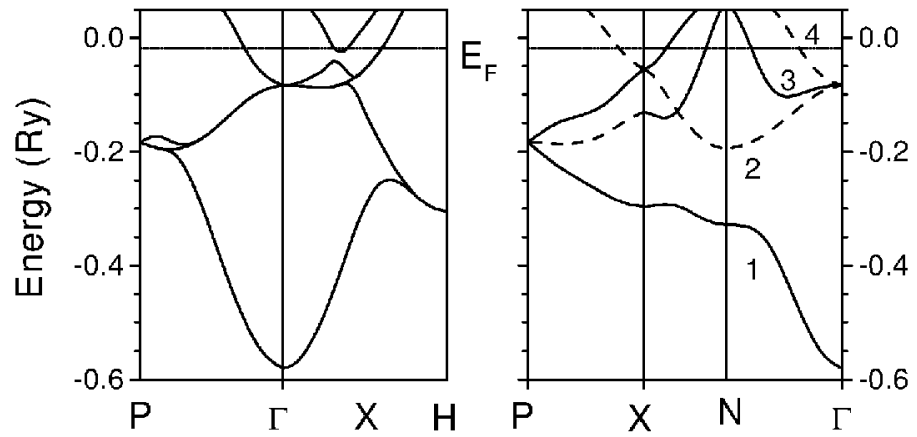

Fig. 1. Band structure of paramagnetic chromium along main crystallographic directions. Bands that do not contribute to the electron and $\mathrm{e}-\mathrm{p}$ momentum densities are marked in the right panel by dashed lines.

In Fig. 1 we present the electron band structure of paramagnetic chromium, calculated within the linear muffin-tin orbital method with the atomic sphere approximation [11]. For the $\Gamma-N$ and $X-N$ directions, the main contribution to $\rho(\boldsymbol{p})$ in the first BZ comes from the first and third bands (marked by 1 and 3 , respectively). The second band (marked by 2 ) does not contribute to $\rho(p)$ for the $\Gamma-N, X-N$, and $X-P$ directions. In Fig. 2 we compare the resulting e-p momentum densities, calculated within various theories for the $X-N$ direction, with the experimental data [4]. Close to the $X$-point only the first band contributes to $\rho(p)$, and all theories give rise to very similar, slightly decreasing, slopes of $\rho(\boldsymbol{p})$. However, the experimental curve shows a little bit sharper structure in this region. In the vicinity of the BZ boundary the contribution of the third band to $\rho(\boldsymbol{p})$ increases, and the differences between the results of all theories become more 

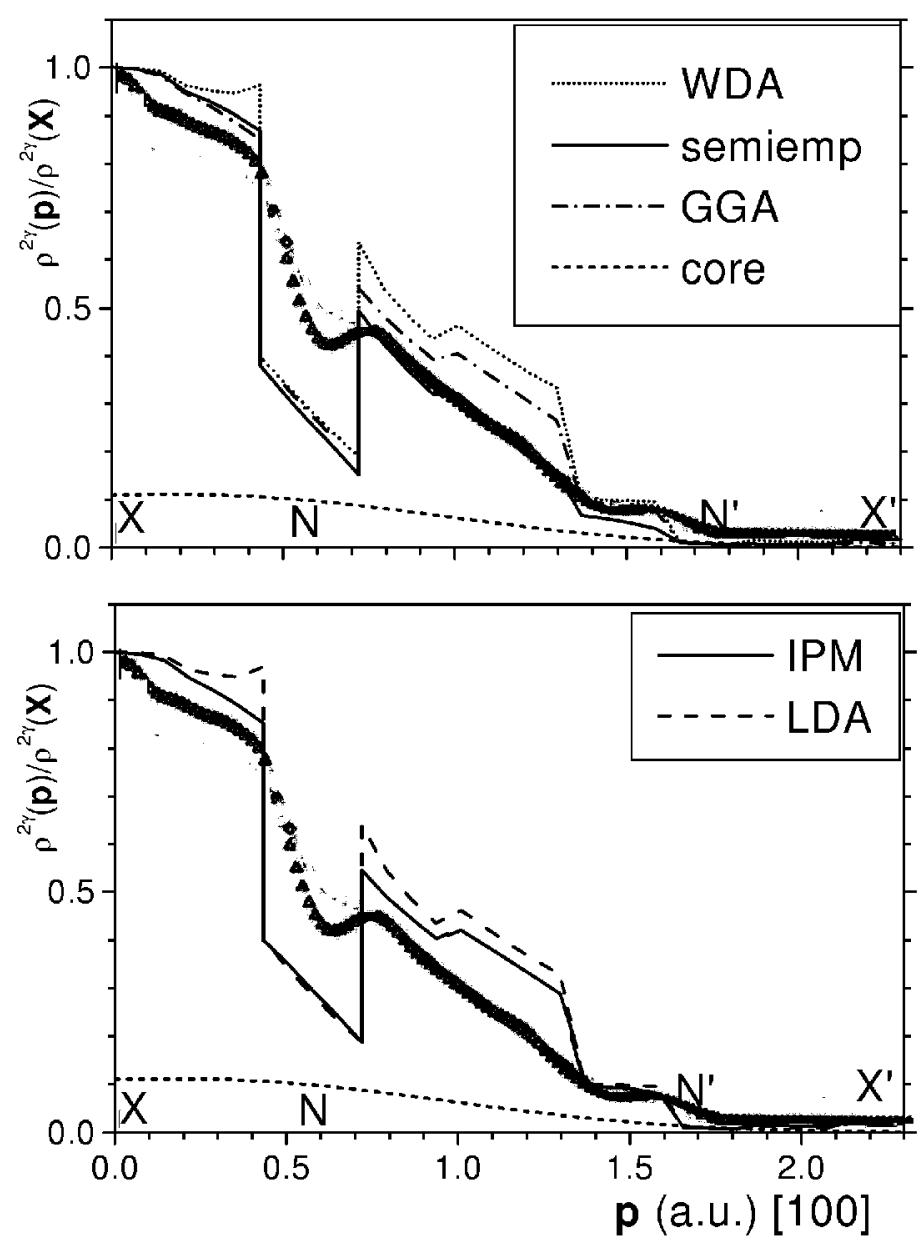

Fig. 2. The e-p momentum densities along the [100] direction (being translated along [001] direction by half of $\Gamma-H$ distance) calculated within various theories compared to experimental data of Ref. [4] (triangles). The spectra are normalised to unity at the $X=[0,0, \pi / a]$ point.

apparent. It seems that the energy dependent approaches of WDA and LDA overestimate the respective $e^{-}-\mathrm{p}$ correlation functions due to the third band, following the strongly increasing energy eigenvalues, $E_{\boldsymbol{k} j}$, in the vicinity of the Fermi energy $E_{\mathrm{F}}$. This effect is well illustrated in Figs. 3 and 4 , where the corresponding e-p enhancement factors are presented. Close to the $N$-point, all the spectra show very similar structures. In the high momentum region it is the semi-empirical curve that provides the best agreement with experiment.

In summary, Figs. 2-4 show that the non-local e-p interaction effects treated within the WDA alter the $\mathrm{e}-\mathrm{p}$ enhancement factors quite substantially, in comparison with LDA. However, the energy dependence arising from both of these 


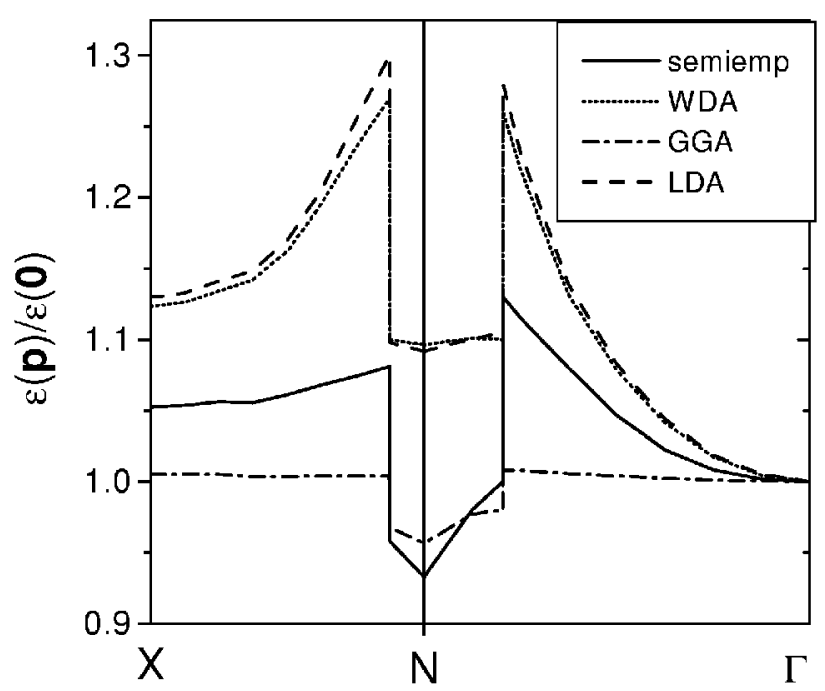

Fig. 3. Total e-p enhancement factor, $\varepsilon(\boldsymbol{p})=\rho(\boldsymbol{p}) / \rho^{\mathrm{IPM}}(\boldsymbol{p})$, calculated along the $\Gamma-N$ and $X-N$ directions within various theories.
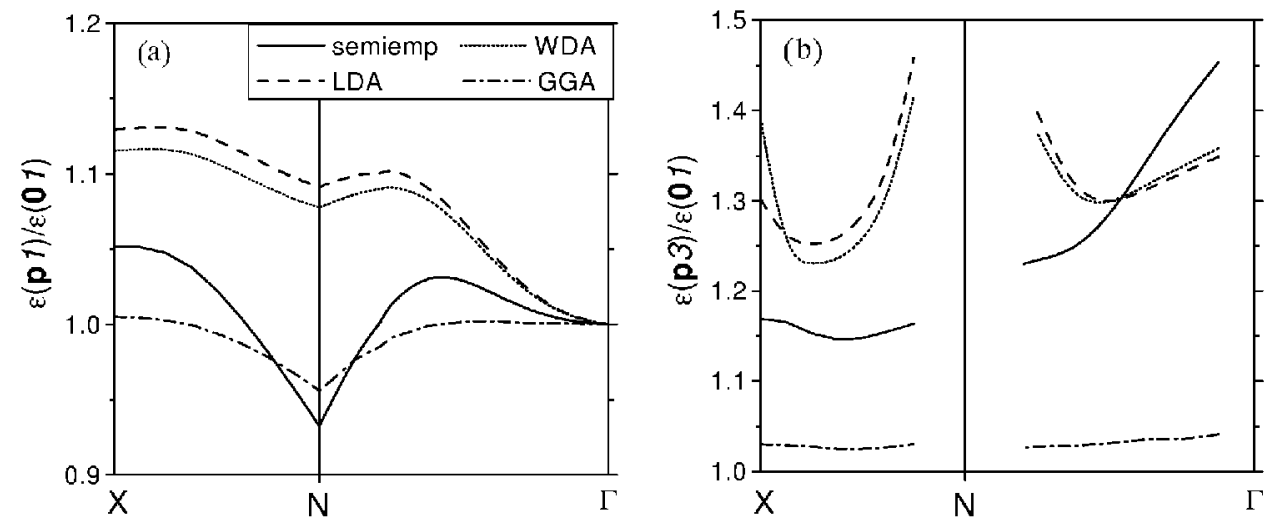

Fig. 4. The e-p enhancement factors, $\varepsilon(\boldsymbol{p} j)=\rho_{j}(\boldsymbol{p}) / \rho_{j}^{\mathrm{IPM}}(\boldsymbol{p})$, for the (a) first and (b) third annihilation active bands, calculated along the $\Gamma-N$ and $X-N$ directions within various theories.

approaches seems to be overestimated, especially for the upper band, in the neighbourhood of the Fermi momentum. Therefore, further improvements of the LDA and WDA are necessary, and will become a subject of future study.

\section{Acknowledgments}

We are grateful to the Committee for Scientific Research (Poland), grant No. 2P03B 10716 for financial support of the work. 


\section{References}

[1] N. Shiotani, T. Okada, H. Skizawa, S. Wakoh, Y. Kubo, J. Phys. Soc. Japan 43, 1229 (1979); C. Bull, A. Alam, N. Shiotani, A.K. Singh, R.M. Singru, in: Positron Annihilation, Eds. P.C. Jain, R.M. Singru, K.P. Gopinathan, World Sci., Singapore 1985, p. 266.

[2] A.K. Singh, A.A. Manuel, E. Walker, Europhys. Lett. 6, 67 (1988); M. Matsumoto, S. Wakoh, J. Phys. Soc. Japan 55, 3498 (1986).

[3] T. Kubota, H. Nakashima, H. Kondo, S. Tanigawa, Mater. Sci. Forum 105-110, 791 (1991); Phys. Status Solidi B 168, 179 (1991).

[4] S.B. Dugdale, Ph.D. thesis, University of Bristol, Bristol 1996; S.B. Dugdale, H.M. Fretwell, M.A. Alam, T. Jarlborg, G. Santi, R.M. Singru, V. Sundararajam, M.J. Cooper, J. Phys., Condens. Matter 10, 10367 (1998).

[5] P. Genoud, Ph.D. thesis, Geneva University, Geneva 1990.

[6] H.M. Fretwell, S.B. Dugdale, R.J. Hughes, J. Brader, M.A. Alam, A. Rodriguez-Gonzalez, J. Phys., Condens. Matter 10, 10375 (1998).

[7] S. Berko, in: Positron Solid State Physics, Eds. W. Brandt, A. Dupasquier, NorthHolland, Amsterdam 1983, p. 64; R.N. West, in: Positron Spectroscopy of Solids, Eds. A. Dupasquier, A.P. Mills, IOS Press, Amsterdam 1995, p. 75.

[8] A. Rubaszek, Z. Szotek, W.M. Temmerman, Phys. Rev. B 58, 11285 (1998); ibid., 61, $10100(2000)$.

[9] S. Daniuk, G. Kontrym-Sznajd, A. Rubaszek, H. Stachowiak, J. Mayers, P.A. Walters, R.N. West, J. Phys. F 17, 1365 (1987).

[10] B. Barbiellini, M.J. Puska, T. Korhonen, A. Hajru, T. Torsti, R.M. Nieminen, Phys. Rev. B 53, 16201 (1996); M. Alatalo, H. Kauppinen, M.J. Puska, K. Saarinen, J. Makinen, P. Hautojärvi, R.M. Nieminen, ibid. 54, 2397 (1996).

[11] O.K. Andersen, Phys. Rev. B 12, 3060 (1975); W.R.L. Lambrecht, O.K. Andersen, ibid. 34, 2439 (1986). 